\title{
Together we can build a smart charging ecosystem
}

Frank Geerts, Baerte de Brey, ElaadNL, The Netherlands

This manuscript was not available on completion of this publication. Thank you for your understanding. 